\title{
Sindbis Virus Reconstruction at $11 \AA ̊$ Resolution Reveals Details of Glycoprotein and Nucleocapsid Organization
}

\author{
W. Zhang, S. Mukhopadhyay, S. V. Pletnev, R. J. Kuhn, M. G. Rossmann and T. S. Baker \\ Department of Biological Sciences, Purdue University, West Lafayette, IN 47907-1392
}

Sindbis virus (SINV) is one of 26 members of the alphavirus genus (family Togaviridae), all of which are enveloped, positive strand RNA viruses.[1] The 52MDa SINV contains an 11.8kb genome, a host-derived lipid membrane, and three s tructural proteins: glycoproteins E1 and E2, and nucleocapsid protein (NCP). Because no high resolution crystallographic analysis of any alphavirus virion has yet been successful, structural investigations of alphaviruses have primarily been performed using electron cryo-microscopy (cryoEM) and three-dimensional image reconstruction of viruses [2, 3] and X-ray crystallographic analysis of the NCP [4] and E1 glycoprotein [5] along with atomic modeling of these molecules into cryoEM density maps [2,5]. Bas ed on such studies, alphaviruses have been shown to be spherical, multi -layered structures of $\sim 720 \AA$ diameter with the capsid and glycoproteins organized with $\mathrm{T}=4$ icosahedral quasi -symmetry. The viral genome is encapsidated by 240 NCPs, which extend to a m aximum radius of $\sim 220 \AA$ where they are enveloped by the viral lipid bilayer. The exterior of the virus contains 80 prominent, trimeric spikes, each of which consists of three E1 -E2 heterodimers. E1 and E2 both have C -terminal transmembrane tails and the E2 tail forms one-to-one interactions with NCP which are believed to be important in virus assembly and stability.[6] Recent studies of SINV deglycosylation mutants have shown that alphavirus E1 and E2 proteins adopt markedly different orientations (E1, mo re tangential; E2, more radial) that are consistent with the known functions (E1, fusogenic; E2, cell attachment) of these glycoproteins.[7]

In this study, aimed at defining alphavirus components with improved clarity, we computed a three dimensional reconstruction of SINV deglycosylation mutant E2 -N318Q at 11 A resolution (FIG.1). Virus sample was prepared for cryoEM and images were recorded in a Philips CM200-FEG electron microscope at $38000 \times$ nominal magnification.[8] Micrographs were digitized at a $7 \mu \mathrm{m}$ step size and subsequently were bin -averaged to $14 \mu \mathrm{m}$ pixels, corresponding to $3.68 \AA$ intervals in the specimen. The final reconstruction was computed from 4931 particle images extracted from 27 micrographs recorded at defocus levels ranging between 1.1 and $2.1 \mu \mathrm{m}$.

The crystal structure of the Semliki Forest virus (SFV) E1 glycoprotein ( $52 \%$ sequence identity with SINV E1) was fitted into the SINV 3D reconstruction making use of the known E1 glycosylation sites as constraints. A difference map, computed by subtracting the modeled E1 densities from the SINV reconstruction, provide clues about the location and structure of E2, which appears to have an extended structure quite similar to E1. The E1 and E2 glycoproteins wind around each other and form one $\mathrm{p}$ etal of a trimeric spike. The SINV reconstruction clearly shows pairs of rod-like features with a left -handed twist that tranverse the bilayer. These features are attributed to the E1 and E2 transmembrane helices and were modeled as an $\alpha$-helical coiled-coil (FIG.1C). The crystal structure of SINV NCP [4] fits nicely into the corresponding density of the SINV reconstruction (FIG.1C), but in an orientation quite distinct from that originally determined from a $25 \AA$ resolution reconstruction of Ross River virus [2, 9].

References:

[1] J. H. Strauss and E. G. Strauss, Microbiol. Rev. 58(1994) 491.

[2] R. H. Cheng et al., Cell, 80(1995) 621.

[3] E. J. Mancini et al., Mol. Cell 5(2000)255. 
[4] H. K. Choi et al., Nature 354(1991)37.

[5] J. Lescar et al., Cell 105(2001)137.

[6] T. L. Tellinghuisen et al., Genetic Engineering 23(2001)83.

[7] S. V. Pletnev et al., Cell 105(2001)127.

[8] T. S. Baker et al., Microbiol. Molec. Biol. Reviews 63(1999)862.

[9] We thank R. Ashmore, P. Chipman, and C. Xiao for help and discus sions. Work supported in part by the NIH Program Project Grant to MGR and TSB (AI45976), the NIH grant to RJK (GM56279), a shared equipment grant from NSF to TSB (BIR 9112921), and an instrumentation reinvestment grant from Purdue University to the Purdue Structural Biology faculty.

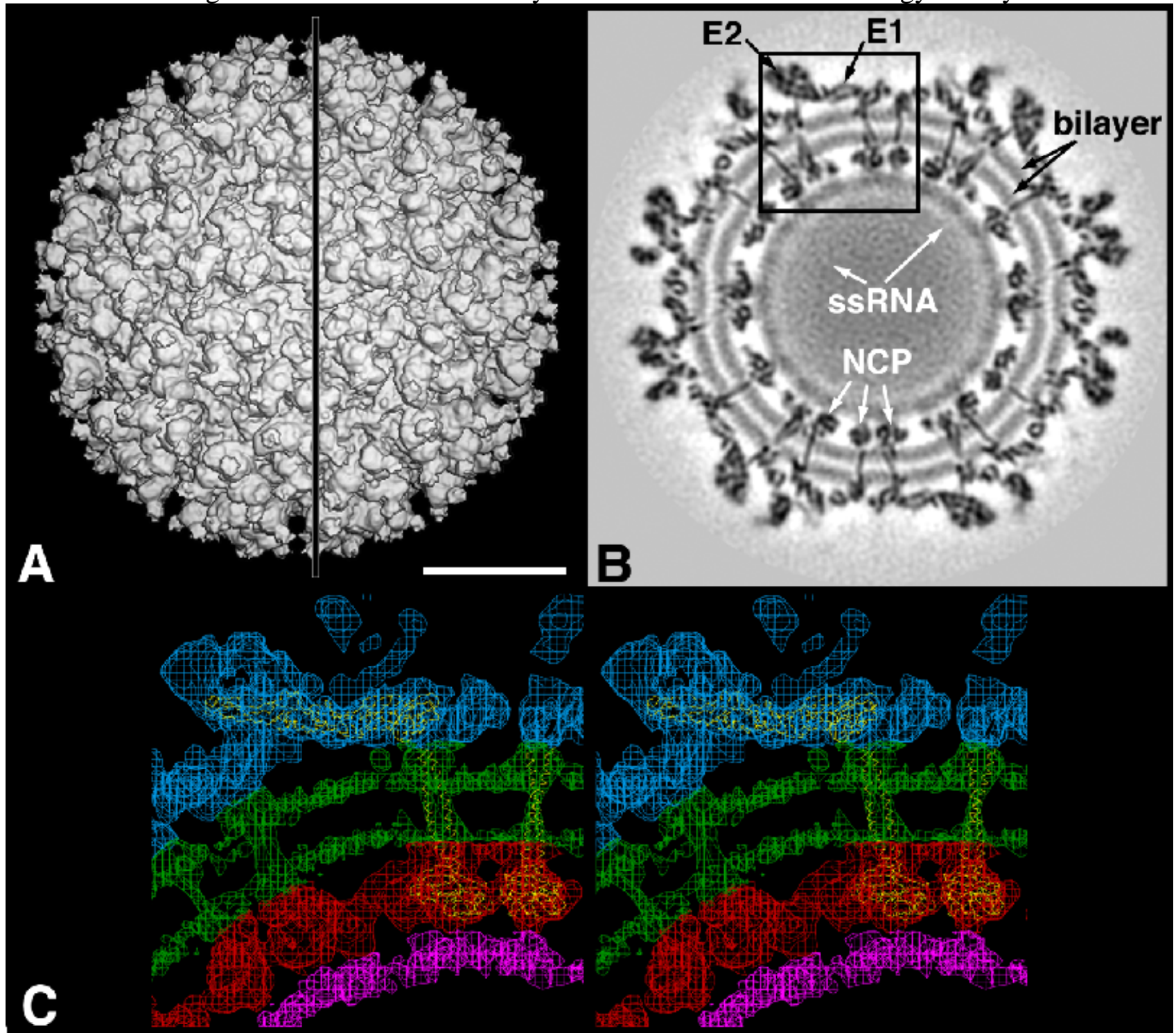

FIG.1 A. Surface-shaded representation of the Sindbis virus N318Q mutant viewed along a 2-fold symmetry axis. B. Cross-section from reconstructed density map (dark line in A) depicts the multilayer organization of SINV (highest density features are darkest). C. Magnified, stereoscopic view of the region outlined by the black square in (B). $\mathrm{C}_{\alpha}$ backbone models of the X-ray structures of SFV E1, general control protein, GCN4, coiled-coil helices, and SINV NCP were fitted into the reconstruction (grey contours). Scale bar $=200 \AA$ for $(A, B)$. 